\title{
Towards a new standard of perioperative fluid management
}

\author{
Conor J Shields \\ Department of Surgery, Mater \\ Misericordiae Hospital, Eccles St, \\ Dublin 7, Ireland
}

\begin{abstract}
Recent studies suggest that current fluid strategies may result in excessive administration of both fluids and electrolytes. Perioperative fluid administration is dictated by an algorithmic approach, taking account of pre-operative deficit, maintenance requirements, and extrapolated third space losses. Salt and water overload is associated with pulmonary edema, ileus, and delayed wound healing. Within an intensive care population, there is a strong correlation between excessive intravascular volume and subsequent mortality, morbidity, and length of stay. Increasing weight has been shown to correspond with mortality, while achieving a negative balance within the first 72 hours of ITU admission has been postulated as an independent predictor of survival. Should a "restricted" rather than a "liberal" perioperative fluid regimen be employed? It is arguable that prevailing fluid therapy is not evidence-based. Recent observations suggest that restraint in fluid administration correlates with better outcome. The development of a protocol-based fluid optimization program may help minimize the risk of perioperative fluid overload.
\end{abstract}

Keywords: perioperative fluid management, restriction, surgical outcome

The aim of perioperative fluid administration is to maintain adequate intravascular volume to ensure acid-base and electrolyte equilibrium, and optimize delivery of oxygen and coagulation factors. Current practices are dictated by an algorithmic approach, taking account of pre-operative deficit, maintenance requirements, and extrapolated third space losses. Recent studies suggest that current strategies may result in excessive administration of both fluids and electrolytes, and that commonly observed postoperative weight gain is a manifestation of the injudicious use of parenteral fluids (Lobo et al 2002; Brandstrup et al 2003). A literature search of the PubMed database for articles meeting the search criteria "perioperative fluid management", "fluid restriction", and "goal-directed fluid therapy" reveals the burgeoning interest in relating various fluid strategies to patient outcome. Between the years 1990 and 1999 there were 8 trials published, rising to 31 trials during the period 2000 to 2007.

The proclivity towards an excess of saline solution in the perioperative period has its origins with the beginnings of modern surgery; anecdotal reports of morbidity associated with excessive intravascular volume emerged shortly after the use of large volume salt solutions became routine (Evans 1911). Writing in the Journal of the American Medical Association in 1911 about "the thoughtless and indiscriminate use of this remedy", Evans wrote: "One cannot fail to be impressed with the danger of... the utter recklessness with which salt solution is frequently prescribed, particularly in the postoperative period..." (Evans 1911). However, the concept of extravascular volume depletion due to "third space" redistribution and evaporative loss, popularized by Shires in the 1960s, lent new vigor to intravenous therapy (Shires et al 1960). The deleterious effects of aggressive resuscitation again became evident during the Vietnam
65 Boulevard Soult, 75012 Paris, France Email conor_shields@hotmail.com 
War, with the emergence of "Danang Lung", now known as acute respiratory distress syndrome (ARDS). However, the use of large volume intravenous salt solutions had become firmly embedded in medical practice.

The consequences of salt and water overload are well established (Lowell et al 1990; Lobo et al 2002). Pulmonary edema compromises gas exchange, and makes the host more susceptible to pneumonia, edema prolongs post-operative ileus, inhibits gastric emptying, and delays feeding, while soft tissue edema reduces lymphatic drainage and local oxygenation, increasing the propensity for delayed wound healing, including anastomotic healing. The excretory load upon the kidney is correspondingly increased, as is ventricular preload, with a potential for decompensation. Within an intensive care population, there is a strong correlation between excessive intravascular volume and subsequent mortality, morbidity, and length of stay (Lowell et al 1990; Alsous et al 2000). Increasing weight, indicative of water and salt retention, has been shown to correspond with mortality (Lowell et al 1990), while achieving a negative balance within the first 72 hours of ITU admission has been postulated as an independent predictor of survival. Alsous and colleagues (2000) noted a significantly better outcome in septic patients with a negative balance of $>500 \mathrm{ml}$ on one or more of the first three days of intensive care.

Six recent prospective randomized trials have addressed the issue of perioperative fluid restriction. Lobo and colleagues (2002) described decreased incidence of postoperative complications, expeditious recovery of gastrointestinal function, and shorter hospital stay, in patients in whom postoperative fluids were restricted to $\leq 2 \mathrm{~L} \mathrm{~d}^{-1}$ crystalloid, when compared with a standard regimen of $\geq 3 \mathrm{~L} \mathrm{~d}^{-1}$. Brandstrup and colleagues (2003) compared two perioperative fluid regimens in172 patients undergoing colorectal resection. In the restricted arm, priority was given to maintaining preoperative weight: preload prior to epidural was omitted, and intraoperative third space losses and diuresis were not replaced. In the postoperative phase, patients received 1L 5\% glucose, and the volume of drain effluent was replaced with $6 \%$ hydroxyethyl starch. The restricted fluid cohort enjoyed significantly less cardiopulmonary and tissue healing complications, without exhibiting any adverse effects. Nisanevich and colleagues (2005) randomized 152 patients undergoing elective abdominal surgery to receive $4 \mathrm{ml}$ or $12 \mathrm{ml} \mathrm{kg}^{-1} \mathrm{hr}^{-1}$, resulting in median perioperative infusates of $1.4 \mathrm{~L}$ and $3.9 \mathrm{~L}$ respectively. The restricted regime was accompanied by a significant reduction in hospital stay with faster return of bowel function, less complications, and a moderated increase in weight (Nisanevich et al 2005).
In contrast, two recent studies reported by Holte and colleagues (2007a, 2007b) failed to show the superiority of a restricted fluid regime when using spirometry as a primary outcome measure in patients undergoing fast-track colonic surgery and knee arthroplasty. Similarly, McKay and colleagues (2006) did not demonstrate any meaningful differences between liberal and restricted fluid regimes, employing hospital stay as the primary endpoint.

Sodium requirements are estimated at $1 \mathrm{mmol} \mathrm{kg}^{-1} \mathrm{~d}^{-1}$, with an accompanying water load of $25-35 \mathrm{~mL} \mathrm{~kg}^{-1}$. Prevailing intraoperative fluid strategies may entail the administration of $10-18 \mathrm{~mL} \mathrm{~kg}^{-1} \mathrm{~h}^{-16}$, with post-operative fluid management consisting of up to $5 \mathrm{~L} \mathrm{~d}^{-1}$ (median $3 \mathrm{~L}$ ), containing up to $770 \mathrm{mmol}$ sodium (median $242 \mathrm{mmol}$ ) (Stoneham and Hill 1997). When IV antibiotics and gastric acid suppressors are included, the fluid load is further exaggerated. Underestimation of the consequences of this salt and water excess may be attributed to dispute over physiological norms and models, and widespread misconceptions regarding daily requirements (Stoneham and Hill 1997; Lobo et al 2001). As a result, measures are not routinely adopted to avoid excessive intravascular volume. Administration of large volumes is further compounded by the lack of scrutiny of fluid prescription, a responsibility frequently delegated to the most junior members of the surgical team.

Should a "restricted" rather than a "liberal" perioperative fluid regimen be employed? Development of clinical guidelines for perioperative fluid management is difficult because of the small number of randomized trials and the influence of perceived wisdom on prevailing practice. Nevertheless, recent observations suggest that restraint in fluid administration correlates with better outcome. Current multimodal optimization pathways facilitate a "restricted" fluid regimen by minimizing the extent of altered fluid distribution in the preoperative patient. As part of accelerated care protocols for gastrointestinal surgery, bowel preparation is less often required; thus preoperative salt and water depletion is less frequent. Ingestion of carbohydrate-rich fluids prior to surgery, with re-institution of oral fluids in the immediate post-operative phase, further diminishes the propensity for iatrogenic dehydration. This reduces the need for epidural preload and facilitates a significant reduction in the volume of perioperative crystalloid required to maintain hemodynamic stability.

The lack of consensus exhibited in recent trials on fluid restriction emphasizes the need for further randomized trials with especial consideration given to primary end points which are likely to accurately reflect improved patient outcome. 
That deficient intraoperative fluid administration leads to suboptimal tissue perfusion is indisputable; that an excess of salt and water may also be deleterious is both intuitive and supported by recent evidence. Perhaps moderation is the wisest course.

\section{References}

Alsous F, Khamiees M, DeGirolamo A, et al. 2000. Negative fluid balance predicts survival in patients with septic shock: a retrospective pilot study. Chest, 117:1749-54.

Brandstrup B, Tonnesen H, Beier-Holgersen R, et al. 2003. Effects of intravenous fluid restriction on postoperative complications: comparison of two perioperative fluid regimens: a randomized assessor-blinded multicenter trial. Ann Surg, 238:641-8.

Evans G. 1911. The abuse of normal salt solution. JAMA, 57:2126-7.

Holte K, Foss NB, Andersen J, et al. 2007a. Liberal or restrictive fluid administration in fast-track colonic surgery: a randomized, double-blind study. Br J Anaesth, 99:500-8.
Holte K, Kristensen BB, Valentiner L, et al. 2007b. Liberal versus restrictive fluid management in knee arthroplasty: a randomized, double-blind study. Anesth Analg, 105:465-74.

Lobo DN, Bostock KA, Neal KR, et al. 2002. Effect of salt and water balance on recovery of gastrointestinal function after elective colonic resection: a randomised controlled trial. Lancet, 359(9320):1812-18.

Lobo DN, Dube MG, Neal KR, et al. 2001. Problems with solutions: drowning in the brine of an inadequate knowledge base. Clin Nutr, 20:125-30.

Lowell JA, Schifferdecker C, Driscoll DF, et al. 1990. Postoperative fluid overload: not a benign problem. Crit Care Med, 18:728-33.

MacKay G, Fearon K, McConnachie A, et al. 2006. Randomized clinical trial of the effect of postoperative intravenous fluid restriction on recovery after elective colorectal surgery. Br J Surg, 93:1469-74.

Nisanevich V, Felsenstein I, Almogy G, et al. 2005. Effect of intraoperative fluid management on outcome after intraabdominal surgery. Anesthesiology, 103:25-32.

Shires T, Brown FT, Canizaro PC, et al. 1960. Distributional changes in extracellular fluid during acute hemorrhagic shock. Surg Forum, 11:115-17.

Stoneham MD, Hill EL. 1997. Variability in post-operative fluid and electrolyte prescription. Br J Clin Pract, 51:82-4. 